\title{
Enhanced Convergence and Robust Performance of Randomized Dynamical Decoupling
}

\author{
Lea F. Santo: * $^{*}$ and Lorenza Viold \\ Department of Physics and Astronomy, Dartmouth College, 6127 Wilder Laboratory, Hanover, NH 03755, USA
}

(Dated: July 6, 2018)

\begin{abstract}
We demonstrate the advantages of randomization in coherent quantum dynamical control. For systems which are either time-varying or require decoupling cycles involving a large number of operations, we find that simple randomized protocols offer superior convergence and stability as compared to deterministic counterparts. In addition, we show how randomization may allow to outperform purely deterministic schemes at long times, including combinatorial and concatenated methods. General criteria for optimally interpolating between deterministic and stochastic design are proposed and illustrated in explicit decoupling scenarios relevant to quantum information storage.
\end{abstract}

PACS numbers: 03.67.Pp, 03.65.Yz, 05.40.Ca, 89.70.+c

Counterintuitive roles of randomness in physical processes have long been acknowledged. A paradigmatic example is stochastic resonance [1], whereby the transmission of a weak signal is maximized at an optimum noise level. Within quantum information science, a number of applications benefiting from randomness in quantum states and operations have been identified recently. Suggestive evidence is offered by dissipation-assisted quantum computation schemes [2] and by the idea that chaos may stabilize quantum algorithms [3]. In the quantum communication setting, the use of random operations has been shown to decrease the communication cost of achieving remote state preparation and of constructing efficient quantum data-hiding schemes [4]. Finally, random unitary operators may pave the way to efficient estimation methods for open quantum systems [5].

In the context of quantum control theory, the fragility of quantum coherence against uncontrolled noise and randomness hindered the exploration of stochastic control methods till recently [6, 7, 8, 9]. General control-theoretic arguments supporting the usefulness of controlled randomness in dynamical decoupling (DD) schemes for switching off unwanted evolution in interacting quantum systems were provided in 7], and have been validated in specific examples [8] (see also [9]). These studies leave, however, several key questions largely unanswered. In particular: For given control resources, under what circumstances (if any) can randomized DD match or outperform the best deterministic schemes known to date? Can randomized design be exploited in general to further push the efficiency and viability of DD schemes?

In this Letter, we present conclusive evidence of the benefits of randomization in light of the above questions. To isolate the factors responsible for different DD performance, we focus on the simplest control benchmark: complete refocusing of Hamiltonian evolution by means of a restricted but otherwise perfect set of control oper-

\footnotetext{
*Electronic address: Lea.F.Dos.Santos@Dartmouth.edu
}

${ }^{\dagger}$ Electronic address: Lorenza.Viola@Dartmouth.edu ations. In the process, we verify conjectures made in [7], stretch the analysis into unexplored domains, and offer criteria for constructing new highly efficient DD protocols. For time-independent systems, we demonstrate the superior averaging of randomized protocols in control scenarios involving sufficiently long control cycles and/or evolution times. For time-varying systems, randomized DD provides a robust strategy in the presence of system uncertainties. The combination of three key ingredients - concatenated control, symmetry, and randomization emerges as a general principle for design optimization.

$D D$ setting.- DD methods modify a target dynamics $H_{0}(t)$ through the addition of a control field $H_{c}(t)$. We assume that $\left\|H_{0}(t)\right\|_{2}=\max \left|\operatorname{eig}\left(H_{0}(t)\right)\right|<\kappa$, for $\kappa>0$ and all $t$. Let $U_{c}(t)=\mathcal{T} \exp \left[-i \int_{0}^{t} H_{c}(u) d u\right](\hbar=1)$ denote the control propagator, with $\mathcal{T}$ indicating time ordering. In a logical frame which explicitly removes $H_{c}(t)$, the controlled evolution is described by the propagator $\tilde{U}(t)=U_{c}^{\dagger}(t) U(t)=\mathcal{T} \exp \left[-i \int_{0}^{t} \tilde{H}(u) d u\right]$, where $U(t)$ is the total propagator in the physical frame and $\tilde{H}(t)=U_{c}^{\dagger}(t) H_{0}(t) U_{c}(t)$ is the logical Hamiltonian [10]. If $H_{0}(t)$ is time-independent and $U_{c}(t)$ periodic with a cycle time $T_{c}$, then $\tilde{U}\left(T_{n}\right)=U\left(T_{n}\right), T_{n}=n T_{c}, n \in \mathbb{N}$, and both frames coincide stroboscopically. The DD objective here is to make $\tilde{U}(T)$, for final time $T>0$, as close as possible to $\mathbb{1}$ according to a metric of choice.

Let the control resources be specified by a discrete set of unitary operators corresponding to a (projective) representation of a group $\mathcal{G}=\left\{g_{j}\right\}, j=0, \ldots,|\mathcal{G}|-1$, in the Hilbert space $\mathcal{H}$ of the system, $\operatorname{dim}(\mathcal{H})=d<\infty$. Both the basic deterministic and random DD protocols may be understood as effecting an appropriate symmetrization of $H_{0}(t)$ according to $\mathcal{G}$. Assume a time-independent system first. In standard deterministic $D D$, average Hamiltonian theory (AHT) allows the logical evolution to be represented as $\tilde{U}\left(T_{n}\right)=\left[\exp \left(-i \bar{H} T_{c}\right)\right]^{n}$, where $\bar{H}=\sum_{k=0}^{\infty} \bar{H}^{(k)}$ and each term $\bar{H}^{(k)}$ is computed from the Magnus expansion [10]. Under the convergence condition $\kappa T_{c}<1$, the leading zero-th order contribution $\bar{H}^{(0)}$ may be mapped into a group average $\bar{H}_{\mathcal{G}}=|\mathcal{G}|^{-1} \sum_{j} g_{j}^{\dagger} H_{0} g_{j}$ by sequentially steering $U_{c}(t)$ through all the group elements $\left\{g_{j}\right\}$ - which corresponds to a train of "bang-bang" 
instantaneous pulses $P_{k}=g_{k} g_{k-1}^{\dagger}, k=1, \ldots,|\mathcal{G}|$, separated by $\Delta t>0$ and with $T_{c}=|\mathcal{G}| \Delta t[11]$. A cyclic protocol based on a fixed, predetermined control path within $\mathcal{G}$ will be referred to as periodic DD, henceforth (PDD), provided first-order averaging is achieved, $\bar{H}^{(0)}=0$. The simplest randomized version of such a DD procedure is obtained by sampling $U_{c}(t)$ over $\mathcal{G}$ uniformly at random according to the Haar measure [7] - leading to what we call nä̈ve random $\mathrm{DD}(\mathrm{NRD})$. Let $\mathbb{E}\{\cdot\}$ denote expectation over all possible control realizations; $\mathcal{G}$-symmetrization still emerges on average at each $t$ via a quantum operation $\mathbb{E}\left\{U_{c}^{\dagger}(t) H_{0}(t) U_{c}(t)\right\}=\bar{H}_{\mathcal{G}}$, and convergence may be rigorously established in the limit $T \Delta t \kappa^{2} \ll 1$ [7].

Lower bounds for the expected logical-frame fidelity of an arbitrary pure state $|\psi\rangle, \mathbb{E}\left\{F_{|\psi\rangle}(T)\right\}=$ $\mathbb{E}\left\{|\langle\psi|\tilde{U}(T)| \psi\rangle|^{2}\right\}$, are given by $\mathbb{E}\left\{F_{|\psi\rangle}(T)\right\} \geq 1$ $\mathcal{O}\left(T^{2} T_{c}^{2} \kappa^{4}\right)$ for PDD, and $\mathbb{E}\left\{F_{|\psi\rangle}(T)\right\} \geq 1-\mathcal{O}\left(T \Delta t \kappa^{2}\right)$ for NRD, respectively [7]. Within their regime of validity, these bounds indicate the potential for NRD to outperform PDD when $|\mathcal{G}|^{2}\left(T \Delta t \kappa^{2}\right) \gg 1$. In order to quantitatively compare DD schemes, a control metric which is both efficiently computable and state-independent is desirable. Here, we consider average (unlike worst-case as in [7]) performance, and remove the dependence upon $|\psi\rangle$ by invoking the (expected) entanglement fidelity [12], evaluated as $\mathbb{E}\left\{F_{e}(T)\right\}=\mathbb{E}\left\{|\operatorname{tr}(\tilde{U}(T)) / d|^{2}\right\} . F_{e}(T)$ is linearly related to the average of $F_{|\psi\rangle}(T)$ over all $|\psi\rangle$. Perfect DD corresponds in this metric to $\mathbb{E}\left\{F_{e}(T)\right\} \rightarrow 1$. In practice, Monte Carlo simulations of the controlled dynamics are used to estimate $\mathbb{E}\left\{F_{e}(T)\right\}$ through a statistical average $\left\langle\left\langle F_{e}(T)\right\rangle\right\rangle$ over control realizations.

Time-independent case: Convergence improvement.By way of illustration, consider $N$ spin- $1 / 2$ particles (qubits), described by the following model Hamiltonian:

$$
H=\sum_{i=1}^{N} \frac{\omega_{i}}{2} \sigma_{i}^{(z)}+\sum_{i<j}^{N} \sum_{a=x, y, z} J_{i j}^{(a)}\left(r_{i j}\right) \sigma_{i}^{(a)} \otimes \sigma_{j}^{(a)},
$$

where $\sigma^{(x, y, z)}=X, Y, Z$ are Pauli operators, and $\omega_{i}, J_{i j}^{(a)}$, denote the frequency of the $i$ th qubit, and the coupling strength of the $i j$ th pair in the $a$ direction, respectively. Heisenberg interactions exponentially decaying with the distance $r_{i j}$ are typical of quantum dot arrays 13, whereas cubic decays describe dipolarly coupled spins in systems ranging from nuclear magnetic resonance (NMR) crystals and liquid-crystals [10, 14] to electrons on Helium [15]. We assume here that $\omega_{i} \approx \omega$, and work in a logical-rotating frame whereby the effective Hamilto$\operatorname{nian} \tilde{H}_{R}(t)=U_{c}^{\dagger}(t) U_{R}^{\dagger}(t)\left[H(t)-\omega \sum_{i} Z_{i} / 2\right] U_{R}(t) U_{c}(t)$, $U_{R}(t)=\exp \left[-i \omega t \sum_{i}^{N} Z_{i} / 2\right]$ [16, 17].

PDD protocols capable of refocusing $H$ for arbitrary parameter values (and in fact, arbitrary interactions of the form $\left.\sigma_{i}^{(a)} \otimes \sigma_{j}^{(b)}\right)$ may be built by recursively nesting DD sequences based on the group $\mathcal{G}_{i}=\left\{\mathbb{1}_{i}, X_{i}, Z_{i}, Y_{i}\right\}$ for each added qubit, $i=2, \ldots, N$ 11, 18, 19]. Although this scheme is not efficient, as the number of pulses per cycle grows as $4^{N-1}$, it allows the effect of large $|\mathcal{G}|$ to

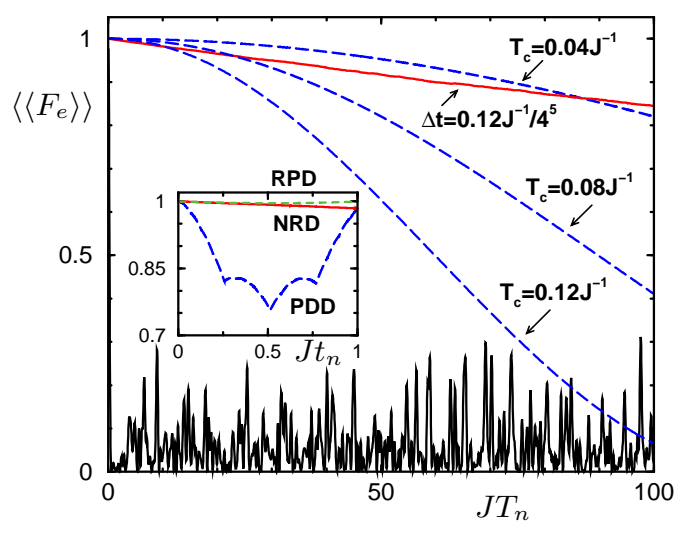

FIG. 1: (color online) PDD (dashed lines) vs. NRD (solid line) based on a nested pulse sequence for $H$ from Eq. (1) in 1D with $N=6, J_{i j}^{(a)} \equiv J|i-j|^{-3}$ in the logical-rotating frame. Main panel: average fidelity at $T_{n}=n|\mathcal{G}| \Delta t$. Free evolution: oscillating solid line. Inset: average fidelity within a cycle, $t_{n}=n \Delta t, \Delta t=10^{-3} J^{-1}$. Dotted line: average over different path choices (RPD, see text). Average over $10^{2}$ realizations.

be studied in moderately small systems. Following [18], the PDD sequence we implement corresponds to a path over the Pauli group $\mathcal{G}^{\mathrm{P}}=\otimes_{i} \mathcal{G}_{i}$ which avoids simultaneous pulses. In NRD, $U_{c}(t)$ is picked uniformly at random over $\mathcal{G}^{\mathrm{P}}$, which may involve collective rotations on up to $R=N-1$. qubits 20]. Numerical results are shown in Fig. 11 As seen in the main panel, the fidelity for NRD decays substantially slower than for PDD. Irrespective of the validity of the short-time condition underlying the lower bounds of [7], these results confirm the faster convergence of stochastic DD when $|\mathcal{G}|$ is large. While $\Delta t$ is kept fixed for NRD, PDD protocols with decreasing $\Delta t$ are considered. NRD eventually surpasses all PDD curves, showing that, for sufficiently long time, the constraints on $\Delta t$ for random DD may be relaxed - which can prove advantageous in practice. Also note that in situations where control constraints make it unfeasible to complete a cycle (inset), NRD outperforms the selected PDD sequence at all intracycle times $t_{n}=n \Delta t<T_{c}-\mathrm{a}$ finding which indicates the existence of better control paths in this time domain.

Time-independent case: Long-time improvement.While different DD protocols are equivalent in the shorttime limit under ideal averaging, performance for finite $\Delta t$ and long time depends critically on the way in which residual errors accumulate. We address this point by extending our comparison to higher-level DD schemes.

In the deterministic domain, two strategies exist for improving over PDD. One, motivated by the Carr-Purcell sequence of NMR, consists of a time-symmetrization of the control path, such that all odd order terms in $\bar{H}$ are also canceled [10, 11]. This leads to symmetric DD (SDD). The other, inspired to NMR iterative techniques [10], implements concatenated DD (CDD) 21]. CDD relies on a temporal recursive structure, the $(\ell+1)$-level pulse sequence being determined by $C_{\ell+1}=C_{\ell} P_{1} C_{\ell} P_{2} \ldots C_{\ell} P_{N}$, 


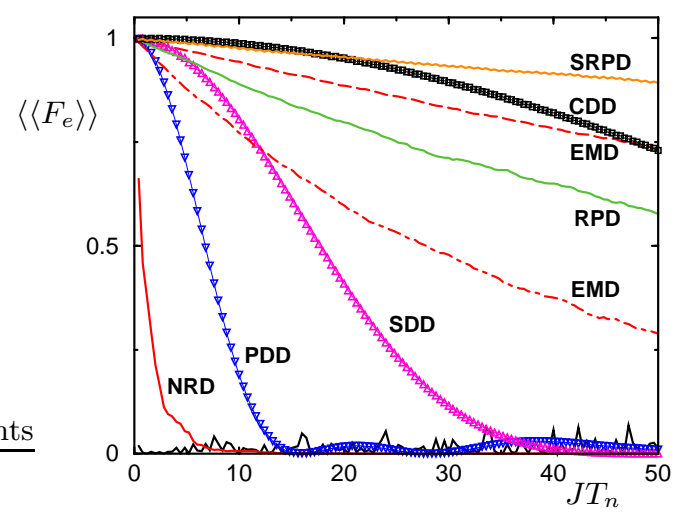

FIG. 2: (color online) Deterministic vs. randomized DD based on $\mathcal{G}_{8}$ for Hamiltonian $H$ as in Fig. 11 except $N=$ $|\mathcal{G}|=8$. Data are averaged over $10^{2}$ control realizations, $\Delta t=0.05 J^{-1}$. Note that at times $T_{n}=8 n \Delta t$, as considered, the inner sequences characterizing SDD, CDD, and SRPD are not necessarily completed. For CDD, $\ell=5$ is achieved at $102.4 J^{-1}$. Dashed and dot-dashed lines correspond to different inner PDD sequences for EMD. Free evolution: (black) solid line.

where $P_{k}$ is the $k$ th pulse, $C_{0}$ is the inter-pulse interval, and $C_{1}$ denotes the generating PDD sequence.

Deterministic DD is plagued by coherent error build-up at long time - as opposed to probabilistic error accumulation in NRD. In order to construct and classify stochastic DD schemes able to ensure good performance at both short and long times, we describe each protocol in terms of an inner and an outer code. The former determines the control path in $\mathcal{G}$, thereby the pulse sequence in the interval $[n,(n+1)]|\mathcal{G}| \Delta t$, subject to the condition that a net effective Hamiltonian with norm $\mathcal{O}(\Delta t)$ is obtained. The outer code specifies additional pulses to be applied at $T_{n}$ according to an outer group $\mathcal{G}^{\prime}$, which need not coincide with $\mathcal{G}$. Combining a fixed PDD sequence with bordering pulses drawn at random from $\mathcal{G}^{\prime}$ leads to embedded DD (EMD), as in [8] with $\mathcal{G}^{\prime}=\mathcal{G}^{\mathrm{P}}$. While choosing $\mathcal{G}^{\prime}$ irreducible typically ensures superior performance, EMD schemes based on reducible $\mathcal{G}^{\prime}=\mathcal{G}$ may still prove useful under restricted control capabilities. However, a potential weakness of EMD is non-uniform performance across the set of $|\mathcal{G}|$ ! inner paths. Path optimization may be shortcut if, at every $T_{n}$, a random path choice is effected - resulting in what we term random path DD (RPD) [7, 22]. Randomization of the inner code ensures that DD performance are robust against path variations. To further improve, control paths which are symmetrized in the same manner as in SDD may be employed, leading to a scheme we call symmetric random path DD (SRPD) 23]. As a final step, interpolated DD protocols, which smoothly switch from high-level deterministic DD (e.g. CDD) to high-level randomized DD (SRPD), allow to optimize control performance over the entire time axis.

Qualitative insight into the above-mentioned DD protocols may be gained through analytical bounds on the expected fidelity decay. For deterministic schemes, we may write $\mathbb{E}\left\{F_{e}(T)\right\} \geq 1-\mathcal{O}\left(T^{2}(|\mathcal{G}| \Delta t)^{2+\alpha} \kappa^{4+\alpha}\right)$, where $\alpha=0,2$ for PDD [7] and SDD [10], respectively. Error bounds for CDD depend on both the system and the concatenation level, but already at $\ell=2 \mathrm{CDD}$ is more efficient than SDD in reducing $\bar{H}^{(k)}, k \geq 2$. Notice, however, that unlike the single-qubit DD analyzed in [21], no guarantee of superpolynomial convergence exists when CDD is based on a reducible group. For randomized DD, the basic error bound is contained in 7] for NRD, further adapted in [8] to EMD. While full derivations will be presented in [17], we find $\mathbb{E}\left\{F_{e}(T)\right\} \geq 1-\mathcal{O}\left(T(|\mathcal{G}| \Delta t)^{3+\alpha} \kappa^{4+\alpha}\right)$, where $\alpha=0$ for EMD and RPD, while $\alpha=2$ for SRPD. As applicability of the above bounds is strictly confined to the regime where $T(|\mathcal{G}| \Delta t) \kappa^{2} \ll 1$, we proceed to a numerical comparison.

For bilinear couplings as in Eq. (11), efficient combinatorial PDD schemes with quadratic complexity are well known 18. First-order DD sequences for up to $N=4^{m}$ qubits, $m \geq 1$, involve $N$ simultaneous pulses. For $N=8$, the basic PDD scheme is derived from the group,

$$
\begin{aligned}
& \mathcal{G}_{8}=\left\{\mathbb{1}, Z_{3} Z_{4} Y_{5} Y_{6} X_{7} X_{8},\right. \\
& Z_{2} Y_{3} X_{4} Z_{6} Y_{7} X_{8}, Z_{2} X_{3} Y_{4} Y_{5} X_{6} Z_{7}, Y_{2} Y_{4} X_{5} Z_{6} X_{7} Z_{8}, \\
& \left.Y_{2} Z_{3} X_{4} Z_{5} X_{6} Y_{8}, X_{2} Y_{3} Z_{4} X_{5} Z_{7} Y_{8}, X_{2} X_{3} Z_{5} Y_{6} Y_{7} Z_{8}\right\} .
\end{aligned}
$$

As $\mathcal{G}_{8}$ is reducible, saturation in the performance of $\mathrm{CDD}$ is observed when $\ell>2$. Numerical results are summarized in Fig. 22 As expected, randomized DD surpasses deterministic DD at long times, the crossing being evident between protocols that have comparable efficiency at short times. The dependence of EMD upon the underlying inner path is very pronounced for this system. Remarkably, CDD is outperformed by SRPD. Although CDD is known to minimize sensitivity to control faults not included here, preliminary results on rotation angle imperfections indicate that the above conclusions remain unchanged.

Exploitation of randomized DD in actual devices will vary depending on whether probabilistic pulse generation capabilities are available or not. In the former case, randomization appears especially promising wherever a large number of experimental runs is required for observation, such as in quantum dot ensemble experiments 24]. Even in the absence of dedicated pulsing capabilities, randomized DD may still be useful (e.g., in solid-state NMR systems) once the procedure is de-randomized, that is, randomization is used off-line to post-select optimal pulse realizations for given physical parameters. Such an option is no longer viable for time-varying control systems.

Time-varying systems and robust performance.- For simplicity, let us specialize Eq. (11) to a situation where only nearest-neighbor couplings are relevant, but a time-dependent anisotropy is effectively present, $J_{i, i+1}^{x, y} \equiv J, J_{i, i+1}^{z}=J \Delta(t)$. For arbitrary $N$, an efficient first-order PDD protocol may be constructed by alternating two collective rotations around perpendicular axes - one acting on all odd qubits, the other on the even ones, e.g., if $N$ is even, $\mathcal{G}=$ $\left\{\mathbb{1}, Z_{1} Z_{3} \ldots Z_{N-1}, Z_{1} Y_{2} Z_{3} Y_{4} \ldots Z_{N-1} Y_{N}, Y_{2} Y_{4} \ldots Y_{N}\right\}$.

While under mild assumptions on the time dependence of $\Delta(t)$, the NRD fidelity bound is still valid []], 


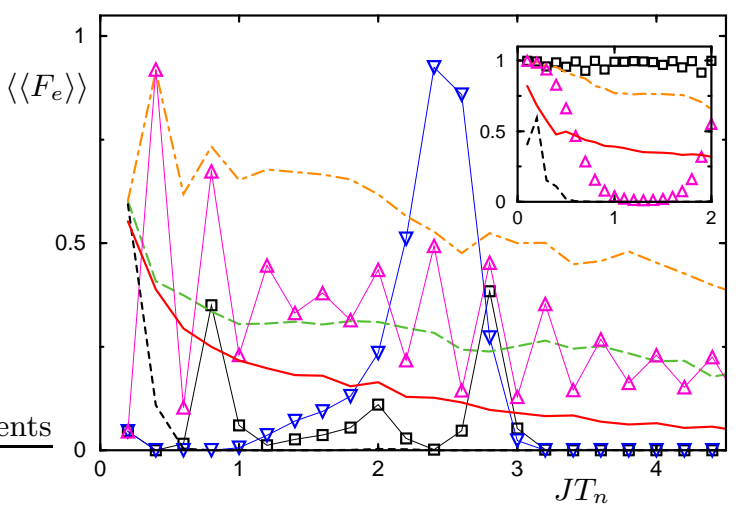

FIG. 3: (color online) Deterministic vs. randomized DD based on a $|\mathcal{G}|=4$ sequence for $1 \mathrm{D}$ nearest-neighbor couplings with $N=8$ in the logical-rotating frame. $\Delta(t)=\sum_{k=1}^{5} \sin \left(10 \pi R_{k} J t\right), R_{k}$ uniformly random in $[0.9,1.1]$. Average fidelity at $T_{n}=n|\mathcal{G}| \Delta t, 10^{2}$ realizations. PDD: down-triangles; SDD: up-triangles, CDD: squares; NRD: solidline; RPD: long-dashed line; SRPD: dot-dashed line. Free evolution: (black) short-dashed line. Main panel: $\Delta t=0.05 \mathrm{~J}^{-1}$, $\tau / \Delta t \approx 2$. Inset: $\Delta t=0.025 J^{-1}-$ notice the sensitivity of deterministic protocols (including CDD) to changes in $\Delta t$.

no general result on deterministic performance is avail- able. Suppose that the system fluctuation entails sign changes over time, with a potentially unknown period $\tau$. Then if $\tau / \Delta t \approx p, p \in \mathbb{N}$, adversarial situations may arise where a pre-established control action is inhibited or even reversed, resulting in surprisingly poor performance of cyclic DD - often worse than NRD or free evolution, see main panel of Fig. 3] Stochastic methods are intrinsically more protected, on average, against such interferences, resulting in more stable DD performance throughout.

Conclusion.- We have shown that randomized decoupling can offer distinctive advantages over deterministic methods in terms of faster convergence, long-time improvement, and robust performance. Together with concatenation and symmetry, randomization provides a versatile toolbox for matching different control needs. Both the analysis of fault-tolerance properties and of the interplay between probabilistic design and robustness deserve a closer scrutiny, also in view of known randomized algorithms for classical control systems 25. It is our hope that the present results will prompt experimental verification in available control devices.

Partial support from Constance and Walter Burke's Special Projects Fund in QIS and from the NSF through grant No. PHY-0555417 is gratefully acknowledged. Thanks to O. Kern for valuable feedback.
[1] L. Gammaitoni et al, Rev. Mod. Phys. 70, 223 (1998).

[2] A. Beige, Inst. Phys. Conf. Ser. 173, 35 (2003).

[3] T. Prosen and M. Žnidarič, J. Phys. A 34, L681 (2001); ibid 35, 1455 (2002).

[4] P. Hayden et al, Commun. Math. Phys. 250, 371 (2004); C. H. Bennett et al, IEEE Trans. Inf. Theory 51, 56 (2005).

[5] J. Emerson et al, Science 302, 2098 (2003).

[6] S. Mancini et al, Europhys. Lett. 60, 498 (2002).

[7] L. Viola and E. Knill, Phys. Rev. Lett. 94, 060502 (2005).

[8] O. Kern and G. Alber, Phys. Rev. Lett. 95, 250501 (2005).

[9] L. F. Santos and L. Viola, Phys. Rev. A 72, 062303 (2005).

[10] U. Haeberlen, High Resolution NMR in Solids: Selective Averaging (Academic Press, New York, 1976).

[11] L. Viola, E. Knill, and S. Lloyd, Phys. Rev. Lett. 82, 2417 (1999); L. Viola, Phys. Rev. A 66, 012307 (2002).

[12] B. Schumacher, Phys. Rev. A 54, 2614 (1996); E. M. Fortunato et al, New J. Phys. 4, 5.1 (2002); M. A. Nielsen, Phys. Lett. A 303, 249 (2002).

[13] D. Loss and D. P. DiVincenzo, Phys. Rev. A 57, 120 (1998).

[14] J. Baugh et al, Phys. Rev. A 73, 022305 (2006).

[15] M. Dykman and P. M. Platzman, Phys. Rev. B 64, 245309 (2001).
[16] Transforming to the rotating frame may be avoided and/ or removing both linear and bilinear terms is possible by slightly generalizing the schemes discussed here [17].

[17] L. F. Santos and L. Viola, in preparation.

[18] M. Stollsteimer and G. Mahler, Phys. Rev. A 64, 052301 (2001).

[19] Highly efficient, size-independent protocols of $\pi / 2$ pulses based on the so-called WAHUHA sequence exist for dipolar DD in solids, corresponding to $J_{i j}^{z} / J_{i j}^{x, y}=-2$ [10].

[20] The fraction with $R \geq N-2$ scales as $3^{N-2}(N+2) / 4^{N-1}$.

[21] K. Khodjasteh and D. A. Lidar, Phys. Rev. Lett. 95, 180501 (2005).

[22] Pseudo-RPD, where paths always begin with $\mathbb{1}$, is able to retain periodic overlap of physical and logical frames, which may be important for stroboscopic signal acquisition. Randomized DD requires, in general, tracking of the applied control trajectory and further frame correction.

[23] After submission of this work, symmetrized EMD has been considered by O. Kern and G. Alber, Phys. Rev. A 73, 062302 (2006).

[24] F. H. L. Koppens et al, Science 309, 1346 (2005); J. R. Petta et al, ibid, 2180 (2005).

[25] R. Tempo, G. Calafiore, and F. Dabbene, Randomized Algorithms for Analysis and Control of Uncertain Systems (Springer, London, 2005). 